\section{§14. Effect of Net Toroidal Current on Measurement of Diamagnetic Beta Value in Heliotron Plasma}

Yamaguchi, T. (Grad Univ.), Watanabe, K.Y., Sakakibara, S., Ida, K.

We study the effect of the net toroidal plasma current on the diamagnetic measurement for large toroidal current experiments in the Large Helical Device (LHD). As a conventional method to compensate the effect of the net toroidal plasma current, the method based on the cylindrical and large aspect ratio plasma model is well known [1]. There the plasma current pass is assumed on the above model geometry. In order to treat precisely the effect, we apply a new method, which evaluates magnetic fluxes of magnetic measurements directly from plasma currents of a MHD equilibrium state with net toroidal current [2]. There is a large difference between the value calibrated by the new method and by a conventional method under the cylindrical and large aspect ratio plasma model because the current pass is quite deferent in the above two methods.

We apply the new calibration method of the diamagnetic flux based on the calculation of 3-D MHD equilibriums with the net toroidal current to the experimental data analysis in order to confirm the validity of the method. Figure 1 shows the time evolution of the acceleration voltage of the neutral bean injectors (NBI), the net toroidal current, the beta value and the line averaged electron density. Here $<\beta_{\text {kin }}>$ is the volume averaged thermal beta value. $\left\langle\beta_{\perp}\right\rangle$ is the perpendicular component of the volume averaged beta value, which is evaluated from the diamagnetic loop flux. One NB is injected in the co-direction during the first half of this discharge $(\sim 4.8 \mathrm{sec})$ and two NB are injected in the ctr-directions during the latter half. Because the main direction of the NB is changed from the co- to the ctr-direction, the toroidal current changes from the co- to the ctr-direction. Reasons for the difference between $<\beta_{\text {kin }}>$ and $(3 / 2)<\beta_{\perp}>$ are considered to be the effect of $\Phi_{\text {Ip }}$ (the flux due to toroidal flux), the flux due to the net toroidal current. Here it is considered that the change of the beam pressure is small during that time because the density and the NBI power are almost constant. The new method is applied to the experimental data during $5.0-6.5 \mathrm{sec}$. $\Phi_{\text {Ip }}$ is evaluated as:

$$
2 \frac{\Phi_{\mathrm{lp}}}{\Phi_{\mathrm{vac}}}=2 \frac{\Phi^{\mathrm{cal}}\left(\beta=\beta_{\mathrm{kin}}, J_{\phi}=J_{\phi}^{\mathrm{MSE}}\right)}{\Phi_{\mathrm{vac}}}-2 \frac{\Phi^{\mathrm{cal}}\left(\beta=\beta_{\mathrm{kin}}, J_{\phi}=0\right)}{\Phi_{\mathrm{vac}}} .
$$

The first term of right hand side is the calculation result of VMEC [3] for the pressure profile evaluated by Thomson scattering and far-infrared interferometers and the toroidal current profile by MSE. The second term of the right hand side shows a calculation result for the measured pressure profile and zero toroidal current on all flux surfaces. Figure 2 shows $<\beta>$ after the calibration of $\Phi_{\text {Ip }}$ using the new method. The calibrated $\langle\beta\rangle$ hardly changes during the period 5.0-6.5 sec unlike before the calibration, and it is 1.5 times larger than that evaluated from the profile measurement, $\left\langle\beta_{\mathrm{kin}}>\right.$. It is thought that the main reason is considered to be the beam pressure.

[1] S. Besshou, C.E. Thomas, T. Ohbata, A. Iiyoshi and K. Uo, Nucl. Fusion 261339 (1986).

[2] T. Yamaguchi T, Plasma Phys. Contr. Fusion to be published in 2006.

[3] S.P. Hirshman et. al., Comput. Phys. Commun. 43143 (1986).

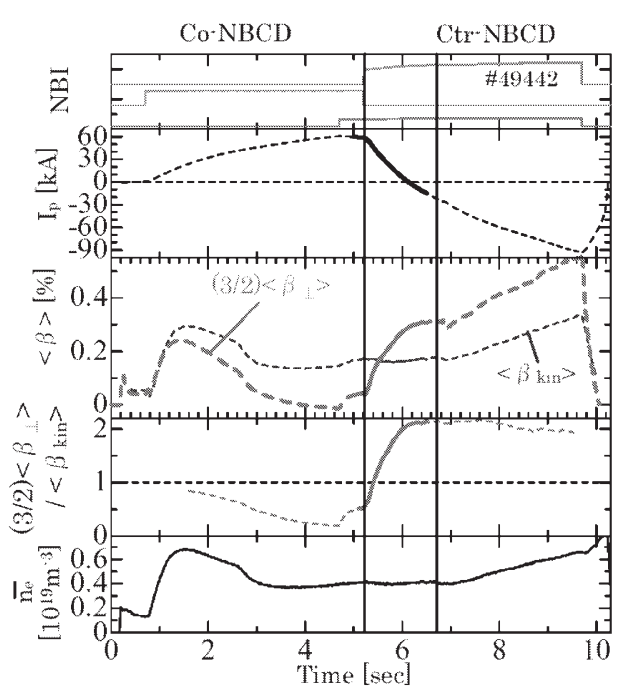

Fig.1 Time evolutions of beta values and some key parameters.

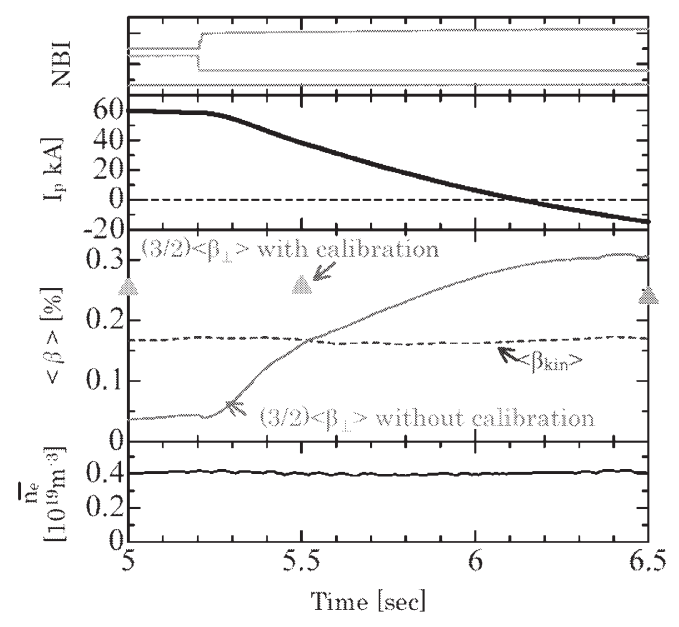

Fig.2 The time evolution of the calibrated beta value after a correction of the net toroidal current effect. 